\title{
A new time-frequency analysis method based on single mode function decomposition for offshore wind turbines
}

\author{
Fushun Liu ${ }^{\mathrm{a}, *}$, Shujian Gao ${ }^{\mathrm{a}}$, Zhe Tian ${ }^{\mathrm{a}}$, Dianzi Liu ${ }^{\mathrm{b}}$ \\ ${ }^{a}$ Shandong Province Key Laboratory of Ocean Engineering, Ocean University of China, Qingdao 266100, China \\ ${ }^{b}$ Engineering, Faculty of Science, University of East Anglia, Norwich, NR4 7TJ, UK
}

\begin{abstract}
The Hilbert-Huang transform (HHT) has been widely applied and recognised as a powerful time-frequency analysis method for nonlinear and non-stationary signals in numerous engineering fields. One of its major challenges is that the HHT is frequently subject to mode mixing in the processing of practical signals such as those of offshore wind turbines, as the frequencies of offshore wind turbines are typically close and contaminated by noise. To address this issue, this paper proposes a new time-frequency analysis method based on single mode function (SMF) decomposition to overcome the mode mixing problem in the structural health monitoring (SHM) of offshore wind turbines. In this approach, the structural vibration signal is first decomposed into a set of window components using complex exponential decomposition. A state-space model is introduced in the signal decomposition to improve the numerical stability of the decomposition, and then a novel window-alignment strategy, named energy gridding, is proposed and the signals are constructed in the corresponding gridding. Furthermore, energy recollection is implemented in each gridding, and the reassembling of these components yields an SMF that is comparable to the intrinsic mode function (IMF) of the HHT, but with a significant improvement in terms of mode mixing. Four case studies are conducted to evaluate the performance of the proposed method. The first case attempts to detect three different frequencies in a simulated time-invariant signal. The second case attempts to test a synthesised signal with segmental time-varying frequencies (each segment contains three different frequencies components). The analysis results in these two cases indicate that mode mixing can be reduced by the proposed method. Furthermore, a synthesised signal with slowly varying frequencies is used. These analysis results demonstrate the effective suppression of non-relevant frequency components using SMF decomposition. In the third case, the experimental data from vortex-induced vibration (VIV) experiments sponsored by the Norwegian Deepwater Programme (NDP) are used to evaluate the proposed SMF decomposition for vibration mode identification. In the final case, field data acquired from an offshore wind turbine foundation
\end{abstract}


and offshore wind turbine are analysed. The mode identification results obtained using SMF decomposition are compared with those produced by the HHT. The comparison demonstrates superior performance of the proposed method in identifying the vibration modes of the VIV experimental and field data.

Keywords: time-frequency analysis, Hilbert-Huang transform, complex exponential decomposition, state-space model, energy gridding

\section{Introduction}

In recent years, offshore wind turbines have been widely applied owing to the stability and reliability of wind energy in a marine environment. Since 2010, the installed capacity has increased by an average of $30 \%$ per year [1]. However, the reliability of offshore wind turbines has become a great source of uncertainty [2]. In the past decade, severe accidents have occurred owing to the break down of wind turbine structures; in particular, there were approximately 1000 accidents in 2014 alone [3, 4]. Furthermore, owing to the high initial installation and maintenance costs, the price of offshore wind energy is considerably higher than that of onshore wind energy [5]. Hence, the ability to assess the structural reliability of offshore wind turbines to prevent unexpected damage/failure has become critical [6]. Although the modal analysis has been successful applied on offshore wind turbines [7], owing to time-varying environmental loadings such as waves, winds and currents, and changes in soil hardness over the lifetime of the structures, their dynamic characteristics change over time which usually requires a selection of appropriate time period before implementing modal analysis. To evaluate the condition of offshore wind turbines, effective signal preprocessing is used to reduce the effects of noises on the accuracy of identification of the modal parameters [8].

Considering the fact of time-varying process of offshore structures, it is often necessary to understand the variation of frequencies with time $[9,10]$, by employing time-frequency analysis methods, which can describe the frequency energy intensity of a signal at different times $[11,12]$. And it is why the time-frequency analysis has been frequently used in the structural health monitoring (SHM) of structures and mechanical systems $[13,14]$. Time-frequency analysis originated from short-time Fourier transform (STFT) analysis, developed in 1946 [15]. STFT assumes that the signal components in a short-time window are stationary. Then, FFT can be used to analyse the power spectrum of the window. Thus, STFT can process nonstationary signals using the concept of window segmentation. However, the fixed window size limits the

\footnotetext{
${ }^{*}$ Corresponding author: percyliu@ ouc.edu.cn (Fushun Liu).
} 
applications of STFT. Following STFT, in 1982, J. Morlet [16] proposed a wavelet transform (WT). WT is a multi-resolution time-frequency analysis method that can solve the contradiction between temporal resolution and frequency resolution. However, WT is limited by fixed wavelet basis functions and suffers from energy leakage in the signal decomposition process. Vladimir et al. [17] used STFT to perform a dynamic analysis to identify the source of vibrations and their interactions in the determination of the contribution of vibration source in a mechanical system. Yu et al. [18] analysed the nonlinear dynamic characteristics of ship rolling in waves using WT. Although these time-frequency analysis methods have contributed considerably to the processing of non-stationary signals, intrinsically, they are Fourier-transform-based methods and can produce contradictory phenomena such as false signals and false frequencies.

In 1998, based on the concept of the intrinsic mode function (IMF), Huang et al. [19] developed the Hilbert-Huang transform (HHT). The so-called empirical mode decomposition (EMD) was proposed to decompose an arbitrary signal into a set of IMFs [20]. By performing Hilbert transform on each IMF, the critical time-frequency characteristics of the signal can be obtained to assess the health condition of a system. Compared with traditional Fourier transform, the HHT can not only deal with non-stationary and transient signals, but also can capture the variation of amplitude and frequency over time. Hence, the adaptability of HHT is better than that of STFT and WT. However, if the frequencies of two or more signal components are close, EMD will lose its decomposition function and the results will suffer from serious mode mixing [21]. In ocean engineering, due to the interaction between wind-wave and structure, the vibration signals of offshore structures are very complex in both time and frequency domains. More importantly, the structural vibration modes are corrupted by strong background noise. Therefore, the performance of HHT for analyzing the practical vibration signals of offshore structures is unsatisfactory. So it is imperative to solve the mode mixing problem. A considerable number of studies have been conducted to address this problem by improving the EMD algorithm [22-24], but because the EMD lacks a strict theory support, the mode mixing problem is still unsolved. The most recent publication attempted to address the theory of EMD [25], but to our best knowledge, little has been applied to offshore wind turbine analysis. Considering the complicated operation condition, it may be not effective to directly apply existing EMD techniques for a vibration analysis of offshore wind turbines. Therefore, it is crucial to develop new effective time-frequency analysis methods that are suitable for offshore wind turbines.

Prony's method was proposed by the French scientist Gaspard de Prony in 1795 [26]. In the Prony method, as periodic assumptions are not required, frequency leakage does not occur; however, in contrast to 
the Fourier transform, it has not been widely used yet. The main reason is that, when using the traditional Prony method, the calculation of the polynomials is an ill-conditioned problem and round-off errors exist for the linear prediction parameters. Consequently, the Prony method is sensitive to noise and is difficult to apply in engineering practice. Based on the Prony method, Hu et al. [27] proposed a signal decomposition method called the Prony-SS method that replaces the high-order homogenous difference equation with a first-order matrix homogenous difference equation, which significantly improved the precision of the decomposition [28]. Compared with the original Prony method, the Prony-SS method demonstrates a superior numerical condition number owing to the use of the state-space model [29]. Moreover, it avoids the sensitivity to noise, sampling frequency, and even truncation error of the Prony method. However, the Prony-SS method is a linear analysis method and it is not applicable when considering the effects of nonlinear factors in the marine environment.

To bridge the research gap in the vibration analysis for offshore wind turbines, a new time-frequency analysis method based on single mode function (SMF) decomposition is proposed. Essentially, the proposed method is a non-stationary generalization of the Prony-SS method using sliding windows. The structural vibration signal is first processed using a sliding window and inside each window, the complex exponentialbased decomposition is performed. Then, the energy gridding method is proposed to implement the energy recollection and reconnect the decomposed window components to obtain the SMFs of the signal. As each SMF is controlled in a predefined frequency interval by the energy gridding, the mode-mixing problem can be reduced significantly. Four case studies are used to evaluate the performance of the proposed method, including a sea test of an offshore wind turbine.

\section{Preliminaries:}

\subsection{Wavelet transform}

The wavelet transform is one of the most widely used traditional methods in the time-frequency analysis for non-stationary signals. By dilating and translating wavelet function $\varphi(t)$, a signal can be decomposed into different frequency components, as

$$
\varphi_{a, b}(t)=\frac{1}{\sqrt{a}} \varphi\left(\frac{t-b}{a}\right)
$$

where $a$ is the scale factor and $b$ is the translation factor. Expanding the function $\varphi(t)$ in an arbitrary square integrable space $L^{2}(R)$ in terms of the functions $\varphi_{a, b}(t)$ results in the continuous wavelet transform (CWT) 
of a signal $x(t)$

$$
W T_{f}(a, b)=<x(t), \varphi_{a, b}(t)>=\frac{1}{\sqrt{a}} \int_{R} x(t) \varphi\left(\frac{t-b}{a}\right) d t
$$

\subsection{Instantaneous frequency}

Frequency is one of the basic concepts in Fourier transform, and it describes a distinct feature of periodic/stationary signals. However, most signals are non-periodic or non-stationary in practical engineering. Carson and Fry [30] first proposed the idea of instantaneous frequency, which is the generalization of traditional frequency but is defined as the derivative of phase angle to time. Van [31] further gave the definition of instantaneous frequency as

$$
\omega(t)=\frac{1}{2 \pi} \frac{d \theta(t)}{d t}
$$

where $\omega(t)$ is instantaneous frequency and $\theta(t)$ is the phase of the signal. As seen in Eq.3, there is only one instantaneous frequency value at each sample point.

The essence of EMD is to decompose any signal into a set of IMFs which should satisfy the following two conditions:

- In the whole data set, the number of extrema (maxima and minima) and the number of zero crossings must either be equal or differ at most by one;

- At any point, the mean value of the envelope defined by local maxima and the envelope defined by the local minima is zero.

Each IMF is actually a narrow-band signal, but with a unique instantaneous frequency $2 \pi \omega(t)$ which can be calculated via the Hilbert transform.

\subsection{Empirical mode decomposition}

The key step of HHT is to decompose a signal into a series of IMFs by using the EMD method. For a real-valued signal $x(t)$, the sifting algorithm can be described as follows:

- Step 1: Identify all the local extrema of $x(t)$.

- Step 2: Interpolate between all the local maxima by a cubic spline line as the upper envelope, $e_{\max }(t)$. Repeat the procedure for the local minima to produce the lower envelope, $e_{\min }(t)$.

- Step 3: Compute the local mean value $m_{1}(t), m_{1}(t)=\left[e_{\max }(t)+e_{\min }(t)\right] / 2$. 
- Step 4: Calculate the difference between $x(t)$ and $m_{1}(t), h_{1}(t)=x(t)-m_{1}(t)$

- Step 5: If $h_{1}(t)$ satisfies the conditions of IMF, than $h_{1}(t)$ is the first component of $x(t)$; If not, $h_{1}(t)$ is treated as the original signal and repeat the process from Step1.

Finally, a criterion based on two thresholds $\theta_{1}$ and $\theta_{2}$ are introduced to control the stopping of the screening process [34]:

$$
\sigma(t)=\frac{\left|e_{\max }(t)+e_{\min }(t)\right|}{\left|e_{\max }(t)-e_{\min }(t)\right|}
$$

So that the shifting is iterated until $\sigma(t)<\theta_{1}$ for some prescribed fraction $1-\alpha$ of the total duration, while $\sigma(t)<\theta_{2}$ for the remaining fraction. In the following calculation, we set default values as $\alpha=0.05, \theta_{1}=$ $0.05, \theta_{2}=0.5$.

At last, the original signal $x(t)$ can be written in terms of the $\operatorname{IMF} h_{i}(t)$ and the residue $r(t)$ as

$$
x(t)=\sum_{i=1}^{n} h_{i}(t)+r(t)
$$

After the decomposition, the Hilbert spectrum can be obtained by using the Hilbert transform on each IMF.

\section{Time-frequency analysis method based on state-space model and energy gridding}

As EMD suffers from mode mixing, it is more effective to replace the IMF decomposition using a suitable decomposition method in the HHT to solve the mode mixing problem. Based on this idea, this paper proposed a new time-frequency analysis method based on the SMF decomposition and Hilbert transform methods.

\subsection{Signal representation using a complex exponential function}

One approach to addressing a nonlinear signal is to divide it into small segments, and in each segment, consider the signal component to be linear. Then, the traditional linear analysis method can be applied to each signal component. Inspired by this linearisation strategy, this paper proposes a non-stationary generalization method based on the Prony-SS method using sliding windows for the vibration signals of offshore wind turbines. The vibration signal is first segmented by a sliding window (contains $N_{k}$ points). In the window, the signal segment is decomposed by a complex exponential-based method. Then, the window slides ahead to decompose the next signal segment. The sliding step size is set as one point to ensure the 
continuity of the signal and to preserve its time-varying properties. Let us assume $N_{m}$ total segments in the window processing, where each has the following form:

$$
y_{m}(t)=\sum_{n=1}^{p_{m}} \gamma_{m, n} e^{\lambda_{m, n} t}
$$

where $y_{m}$ is the $m$ th segment, $m=1,2, \ldots, N_{m}$. The sampling interval is set to $\Delta t, t_{k}=k \Delta t$ and the discrete digital signal $y_{m, k} \equiv y_{m}\left(t_{k}\right)$, where $k=0,1, \ldots, N_{k}-1$ can be expressed as

$$
y_{m, k}=\sum_{k=1}^{p_{m}} \gamma_{m, n} z_{m, n}^{k}
$$

where $p_{m}$ is the number of terms and $z_{m, n}^{k}=e^{\lambda_{m, n} k \Delta t}$. Because $y_{m}(t)$ is a real-valued signal, $\lambda_{m, n}$ must be real numbers or occur in complex conjugate pairs, and the coefficients $\gamma_{m, n}$ corresponding to $\lambda_{m, n}$ must also appear in real numbers or complex conjugate pairs. Let $\gamma_{m, n} \equiv A_{m, n} e^{i \theta_{m, n}}$, where $A_{m, n}$ is the amplitude and $\theta_{m, n}$ is the initial phase, $\lambda_{m, n}=-\alpha_{m, n}+i \omega_{m, n}$, where $\alpha_{m, n}$ is the damping factor in seconds ${ }^{-1}$ and $\omega_{m, n}$ is the frequency in radians.

\subsection{Estimation of parameters in SMF}

In Eq.6, the exponential functions $e^{\lambda_{m, n} t}$ form a basis on the open interval $0<t<T$ [32]. The Prony series (Eq.6) can be evidently viewed as the general solution of a $p_{m}$ th-order homogeneous linear ordinary differential equation with constant real-valued coefficients $a_{m, n}$

$$
\sum_{n=0}^{p_{m}} a_{m, n} y_{m}^{(n)}(t)=0
$$

where $y_{m}^{(n)}(t)=d^{n} y_{m} / d t^{n}$. The characteristic equation of Eq.8 can be written as

$$
\sum_{n=0}^{p_{m}} a_{m, n} \lambda_{m, n}=0
$$

For a discrete digital signal $y_{m, k}\left(k=0,1,2, \ldots, N_{k}-1\right)$, Eq. 8 can be transformed into the $p_{m}$ th-order difference equation

$$
\sum_{n=0}^{p_{m}} b_{m, n} y_{m, k+n}=0 \quad \text { for } \quad k=0,1, \ldots, N_{k}-p_{m}-1
$$

where $b_{m, n}$ represents real-valued constants. Without the loss of generality, let $b_{m, p_{m}}=1$. Then, the characteristic equation corresponding to Eq. 8 can be expressed as

$$
\sum_{n=0}^{p_{m}} b_{m, n} z_{m}^{n}=0
$$


When Eq.8 is directly solved, an ill-conditioned problem can occur. To address this problem, the high-order difference equation is reduced to a first-order matrix difference equation. Then, the solution is obtained by solving the eigenvalue problem of the matrix [27]. Let us define

$$
x_{m, 1}=y_{m}^{(0)}, x_{m, 2}=y_{m}^{(1)}, \ldots, x_{m, p_{m}}=y_{m}^{\left(p_{m}-1\right)}
$$

Then, assuming $a_{m, p_{m}}=1$, Eq. 8 can be rewritten as a first-order matrix difference equation

$$
\dot{\mathbf{x}}_{m}(t)=\digamma_{m} \mathbf{x}_{m}(t)
$$

where

$$
\begin{aligned}
\dot{\mathbf{x}}_{m}(t) & =\frac{d \mathbf{x}_{m}(t)}{d t} \\
\digamma_{m} & =\left[\begin{array}{ccccccc}
0 & 1 & 0 & 0 & \ldots & 0 & 0 \\
0 & 0 & 1 & 0 & \ldots & 0 & 0 \\
0 & 0 & 0 & 1 & \ldots & 0 & 0 \\
\vdots & \vdots & \vdots & \vdots & \ddots & \vdots & \vdots \\
0 & 0 & 0 & 0 & \ldots & 0 & 1 \\
-a_{m, 0} & -a_{m, 1} & -a_{m, 2} & -a_{m, 3} & \ldots & -a_{m, p_{m}-2} & -a_{m, p_{m}-1}
\end{array}\right] \in \mathbb{R}^{p_{m} \times p_{m}}
\end{aligned}
$$

and

$$
\mathbf{x}_{m}(t)=\left[\begin{array}{llll}
x_{m, 1} & x_{m, 2} & \cdots & x_{m, p_{m}}
\end{array}\right] \in \mathbb{R}^{p_{m}}
$$

For certain values of $\mathbf{v}_{m}$ and $\lambda_{m}$, all the solutions of Eq.13 have the form $\mathbf{x}_{m}(t)=\mathbf{v}_{m} \mathrm{e}^{\lambda_{m} t}$. Substituting this into Eq.13 yields

$$
\digamma_{m} \mathbf{v}_{m}=\lambda_{m} \mathbf{v}_{m}
$$

Theoretically, the $p_{m}$ eigenvalues of the matrix $\digamma_{m}$ are exactly the $p_{m}$ roots of the characteristic equation of Eq.9 [33]. In the Prony's method, the roots of Eq.9 would be determined. However, this is a typical ill-conditioned problem. Hence, herein, a low-order state-space model is introduced to compute the eigenvalues of the matrix $\digamma_{m}$ to avoid calculating the roots of Eq.9.

The eigenvalue analysis is performed on matrix $\digamma_{m}$, and then a Hankel matrix is introduced as follows:

$$
\mathbf{H}_{m}(k)=\left[\begin{array}{cccc}
y_{m, k} & y_{m, k+1} & \cdots & y_{m, k+\eta-1} \\
y_{m, k+1} & y_{m, k+2} & \cdots & y_{m, k+\eta} \\
\vdots & \vdots & \ddots & \vdots \\
y_{m, k+\xi-1} & y_{m, k+\xi} & \cdots & y_{m, k+\eta+\xi-2}
\end{array}\right]
$$


where $\xi$ and $\eta$ are the selected number of row and column of the Hankel matrix, respectively.

Considering $k=0$ and applying the singular value decomposition to $\mathbf{H}_{m}(0)$, we obtain

$$
\mathbf{H}_{m}(0)=\left[\begin{array}{ll}
\mathbf{U}_{m, 1} & \mathbf{U}_{m, 2}
\end{array}\right]\left[\begin{array}{cc}
\mathbf{S}_{m, 1} & \mathbf{0} \\
\mathbf{0} & \mathbf{0}
\end{array}\right]\left[\begin{array}{c}
\mathbf{V}_{m, 1}^{\mathrm{T}} \\
\mathbf{V}_{m, 2}^{\mathrm{T}}
\end{array}\right]=\mathbf{U}_{m, 1} \mathbf{S}_{m, 1} \mathbf{V}_{m, 1}^{\mathrm{T}}
$$

In theory, the number of non-zero singular values in Eq.19 is equal to the model order of the system, $p_{m}$, that is, $\mathbf{U}_{m, 1} \in \mathbb{R}^{\xi \times p_{m}}, \mathbf{S}_{m, 1} \in \mathbb{R}^{p_{m} \times p_{m}}$ and $\mathbf{V}_{m, 1} \in \mathbb{R}^{\eta \times p_{m}}$, and the rank of $\mathbf{H}_{m}(0)$ is $p_{m}$. When the rank of the matrix is exceeded, its singular values should be zero. Letting $k=1$ and $\mathbf{H}_{m}(1)$ can be expressed as

$$
\mathbf{H}_{m}(1)=\mathbf{U}_{m, 1} \mathbf{S}_{m, 1}^{1 / 2} \mathbf{A}_{m} \mathbf{S}_{m, 1}^{1 / 2} \mathbf{V}_{m, 1}^{\mathrm{T}}
$$

Then, a realization of system of the matrix is achieved as

$$
\boldsymbol{A}_{m}=\boldsymbol{S}_{m, 1}^{-1 / 2} \boldsymbol{U}_{m, 1}^{\mathrm{T}} \boldsymbol{H}_{m}(1) \boldsymbol{V}_{m, 1} \boldsymbol{S}_{m, 1}^{-1 / 2}
$$

While the computed eigenvalues of $\boldsymbol{A}_{m}$ are $\tilde{z}_{m, n}$, the corresponding $\tilde{\lambda}_{m, n}$ in Eq. 6 are calculated as $\tilde{\lambda}_{m, n}=$ $\ln \left(\tilde{z}_{m, n}\right) / \Delta t$ [27]. Substituting $\tilde{\lambda}_{m, n}$ into Eq.7, the corresponding $\tilde{\gamma}_{m, n}$ can be obtained. Here, the $\tilde{\lambda}_{m, n}$ and $\tilde{\gamma}_{m, n}$ represent the obtained values of $\lambda_{m, n}$ and $\gamma_{m, n}$ after calculation.

\subsection{SMF estimation using energy gridding}

After sliding the window from the beginning to the end, all the decomposition are completed, and the next step is to integrate these decomposed components. To collect these decomposed components, the range of frequency $\left[f_{\min } f_{\max }\right]$ is divided by a frequency interval $\Delta f$. To improve the precision and accuracy of the results, $\Delta f$ can be minimised according to the accuracy required. After dividing the range of frequency, these frequency intervals and the sliding window will divide the entire time-frequency space into a special gridding that we named energy gridding. By using the obtained $\tilde{\lambda}_{m, n}$, a bridge can be established between the decomposed components and their corresponding energy gridding.

Using Eq. 6, the reconstruction can be completed in each gridding, and owing to the sliding step set to be one, at each point, the step of reconstruction has been repeated several times. Then, a process of energy recollection, which averaging the reconstructed components $\tilde{y}_{q}^{[m, l]}(t)$ from different segments but at the same time, is performed to get a new reconstructed signal in each frequency bin as

$$
y^{[m, l]}(t)=\frac{\sum_{q=1}^{N^{[m, l]}} \tilde{y}_{q}^{[m, l]}(t)}{N^{[m, l]}}
$$

where $q$ is the number of decomposed components from different segments but at the same time in one frequency interval, $[m, l]$ represents the gridding divided by the $l$ th frequency bin and the $m$ th segment, 
$N^{[m, l]}$ is the number of components that fall into the gridding. $\tilde{y}_{q}^{[m, l]}(t)$ and $y^{[m, l]}(t)$ represent the $q$ th reconstructed signal and the obtained signal after averaging in corresponding gridding, respectively.

Then, the segmented signals in each frequency bin can be concatenated as

$$
s_{j}^{[m, l]}(t)=\sum_{m=1}^{N_{m}-1} y^{[m, l]}(t)
$$

where $s_{j}^{[m, l]}$ is the $j$ th SMF constructed by $m$ th segment and $l$ th frequency bin.

In practice, the signal can be decomposed into a set of SMFs and if the frequency interval $\Delta f$ is sufficiently small, the decomposed signal can be regarded as a single frequency component. Then, the original signal can be reconstructed by the sum of SMFs as

$$
y(t)=\sum_{j=1}^{N_{j}} s_{j}^{[m, l]}(t)
$$

where $N_{j}$ is the number of constructed SMFs by Eq.23.

\subsection{Time-frequency analysis using the SMF}

After expressing the original signal as the sum of SMFs, similar to the HHT method, the Hilbert transform is introduced to investigate the time-frequency characteristics of the signal. To make it clear for expression, denote $s_{j}(t)$ as the decomposed SMFs, which means $s_{j}(t)=s_{j}^{[m, l]}(t)$. And performing the Hilbert transform for each SMF $s_{j}(t)$ in the upper form

$$
\widehat{s}_{j}(t)=H\left[s_{j}(t)\right]=\frac{1}{\pi} P V \int_{-\infty}^{\infty} \frac{s_{j}(\tau)}{t-\tau} \mathrm{d} \tau
$$

where $P V$ is the Cauchy principal value of the integral, which is used to solve the singularity at $\tau=t$. Thus, we can construct the analytical signal as

$$
z_{j}(t)=s_{j}(t)+i \widehat{s}_{j}(t)=B_{j}(t) \mathrm{e}^{i \phi_{j}(t)}
$$

where $i=\sqrt{-1}, B_{j}(t)$ is the amplitude function, and $\phi_{j}(t)$ is the phase function.

$$
B_{j}(t)=\sqrt{s_{j}(t)^{2}+\widehat{s_{j}}(t)^{2}}
$$

and

$$
\phi_{j}(t)=\arctan \frac{\widehat{s_{j}}(t)}{s_{j}(t)}
$$


As the SMFs are monocomponent, the time derivative of instantaneous phase $\phi_{j}(t)$ is the instantaneous frequency, i.e. [19]

$$
f_{j}(t)=\frac{1}{2 \pi} \times \frac{d \phi_{j}(t)}{d t}
$$

Thus, the original signal can be expressed as

$$
x(t)=R e \sum_{j=1}^{N_{j}} B_{j}(t) \mathrm{e}^{i \int 2 \pi f_{j}(t) \mathrm{d} t}
$$

This time-frequency distribution of the amplitudes is designated the Hilbert spectrum $H(\omega, t)[36]$ :

$$
H(\omega, t)=R e \sum_{j=1}^{N_{j}} B_{j}(t) \delta\left(\omega, \omega_{j}(t)\right)
$$

where $\omega_{j}(t)=2 \pi f_{j}(t)$ and $\delta$ is the Kronecker delta function which is 1 if the variables are equal and 0 otherwise. The Hilbert spectrum provides joint distribution information on the time and frequency domains and describes the change of the frequency with time. Distinct characteristics of the signal in the time and frequency domains can be obtained from the spectrum to assess the condition of the structure.

\subsection{Scheme of the proposed method}

The proposed time-frequency analysis process can be summarised in three sequential main steps; the flowchart is displayed in Fig. 1:

- Representation of signal using a complex exponential function. A sliding window (the sliding step equals one point) is used to divide the signal into small segments. Then, the poles $\gamma_{m, n}$ and residues $\lambda_{m, n}$ of all segmental signals are estimated by introducing a state-space model.

- Energy gridding to estimate the SMFs. An energy gridding is introduced, and the signals are reconstructed in a corresponding gridding using Eq.6; then, Eq.22 is used to implement the energy recollection in each gridding. Using Eq.23, the signals in each frequency interval are reassembled and the SMFs are generated.

- Time-frequency analysis using the SMFs: After obtaining the expression of the SMFs, the original signal can be reconstructed by a sum of SMFs as in Eq.24; then, the Hilbert transform is implemented on each SMF using Eq.25 and the time-frequency spectrum can be obtained. 


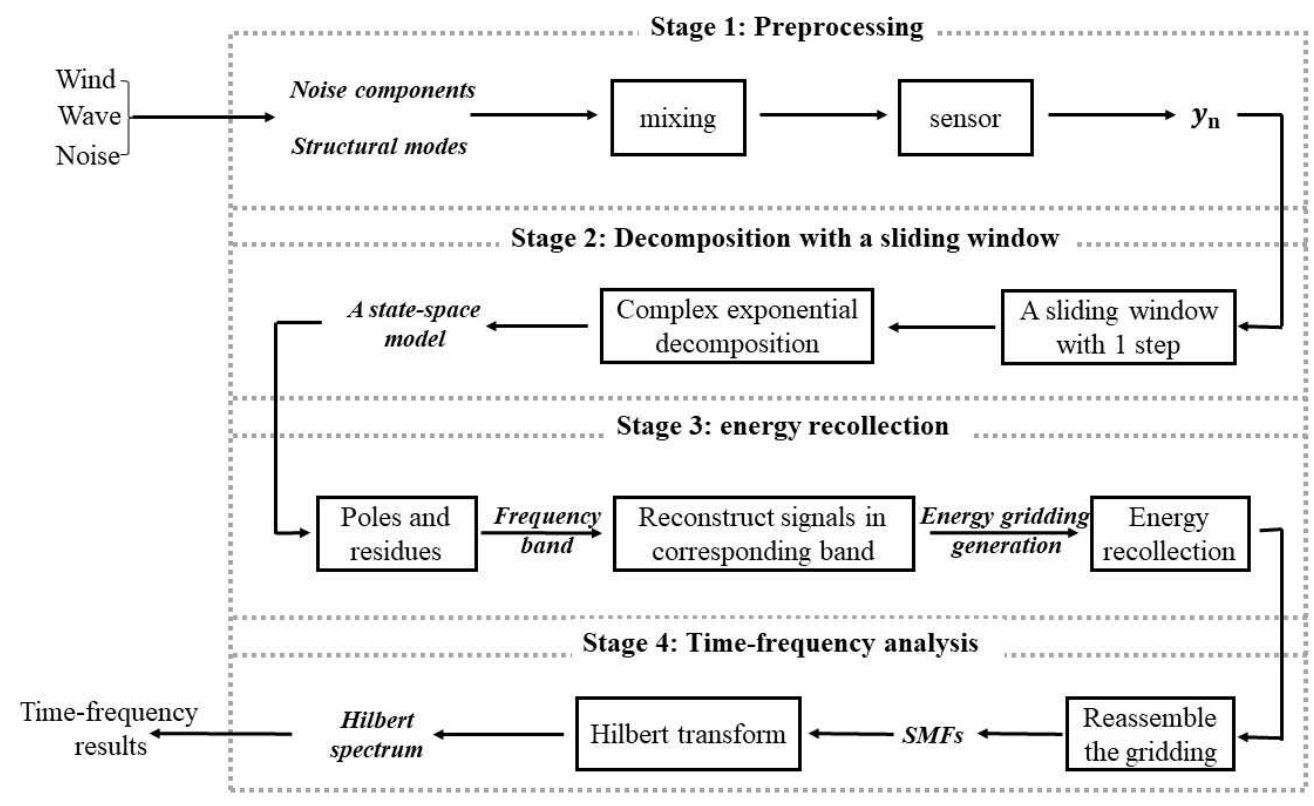

Fig. 1: Flowchart of the proposed method.

\section{Numerical validations}

\subsection{Case study 1: Separating close frequencies}

In this case study, a time-frequency analysis was performed on a signal using the proposed SMF method. The analysed signal contained three close frequency components, as indicated in Eq.32.

$$
y_{k}=\sum_{n=1}^{3} B_{n} e^{\left(-\alpha_{n} t_{k}\right)} \cos \left(2 \pi f_{n} t_{k}+\theta_{n}\right)
$$

The detailed parameters used in Eq.32 are listed in Table 1. As discussed in reference [35], the ratio of frequencies and amplitudes between signals cannot be too close, e.g., for a superimposed signal consisting of two signals (assume the frequencies are $f_{1}(t)$ and $f_{2}(t)$; amplitudes are $A_{1}$ and $A_{2}$ ), the effective decomposition by using the HHT requires: (1) $A_{1} f_{1}(t)>\approx A_{2} f_{2}(t)$ and $3 f_{2}(t)>\approx f_{1}(t)>\approx 2 f_{2}(t)$; or (2) $A_{1} f_{1}(t)>\approx A_{2} f_{2}(t)$ and $f_{1}(t)>\approx 3 f_{2}(t)$. To satisfy the two conditions, in this case, we have selected two sets of signals with frequencies of $8,25,60 \mathrm{~Hz}$ and $12,13,15 \mathrm{~Hz}$ for experimentation. The first one was used to verify the ability of the proposed method to process the time-invariant signals, and the second one was used to investigate the mode mixing problem. 
Table 1: Detailed parameters used in Eq.32

\begin{tabular}{c|ccccc}
\hline$n$ & $f_{n}($ Scenario $)$ & $f_{n}($ Scenario 2$)$ & $B_{n}$ & $\theta_{n}$ & $\alpha_{n}$ \\
\hline 1 & 8 & 12 & 0.2 & $\pi / 4$ & 0.02 \\
\hline 2 & 25 & 13 & 0.6 & $-\pi / 4$ & 0.01 \\
\hline 3 & 60 & 15 & 0.3 & $\pi / 6$ & 0.005 \\
\hline
\end{tabular}
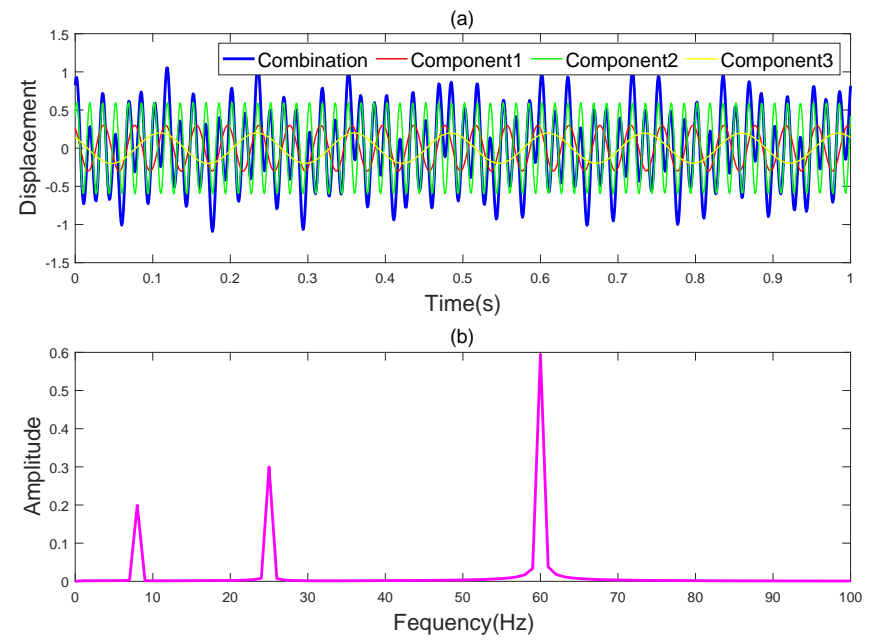

Fig. 2: Time and frequency plots of the original signal in Scenario 1: (a) waveforms of the original signal and (b) amplitude spectrum of the original signal.

\subsubsection{Scenario 1: Time-invariant signal with 8, 25 and $60 \mathrm{~Hz}$}

From Table 1, it can be observed that three frequencies $(8,25$, and $60 \mathrm{~Hz})$ were used for the signal. Fig. 2 (a) displays the original signal and its three components in time domain. As the signal was time-invariant, the frequencies did not change throughout the time period. In the simulation, the sampling frequency was 1 $\mathrm{kHz}$, and the number of data points $N$ was 1000 . Fig. 2 (b) depicts the amplitude spectrum of the original signal.

The proposed SMF decomposition was then performed on the original signal. As the signal was timeinvariant, the proposed complex exponential decomposition was used directly for the entire period [37] by setting the window length equal to the signal length. As there were three frequency components at each time, the model order was set to 6, and $\Delta f$ was set to 0.1. The realisation $\boldsymbol{A}_{m}$ was obtained using Eq.21, and then the poles $\lambda_{m, n}$ and residues $\gamma_{m, n}$ were obtained to reconstruct the signals in the corresponding windows using Eq.6. By repeating the signal decomposition, and the energy recollection can be imple- 

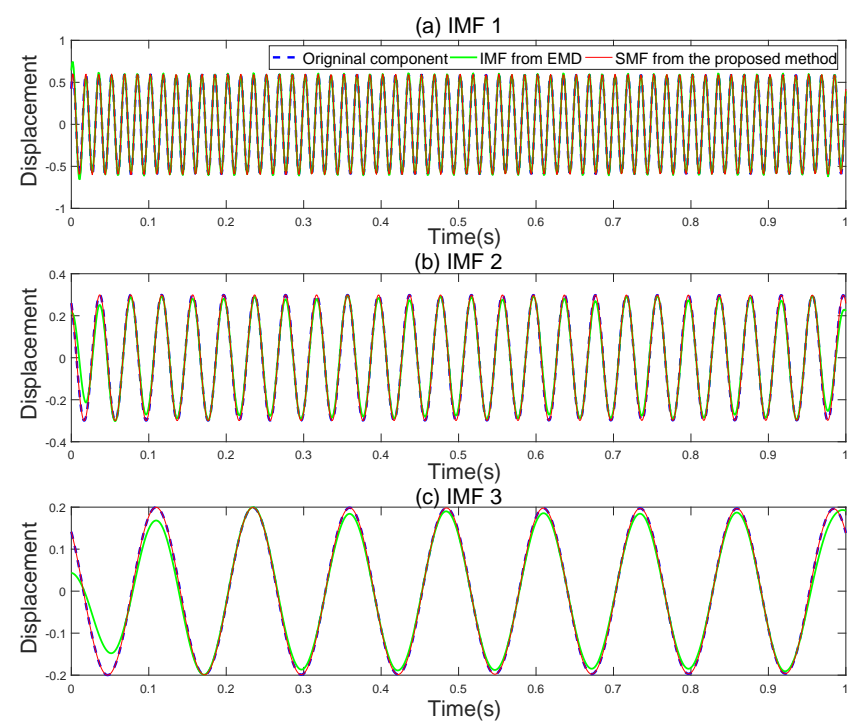

Fig. 3: Comparison between IMFs, SMFs and the original components: (a) IMF1, (b) IMF2, and (c) IMF3.

mented by Eq.22. Then by reassembling the process for the exponential window, and finally splicing all the components using Eq.23, a set of SMFs was obtained. Fig. 3 compares the decomposition results between the IMFs and SMFs. It can be observed that the SMFs could identify the original three frequency components accurately. Furthermore, it can be observed in Fig. 3 that the IMFs successfully tracked the original signal components; however, at both ends, the tracking performance was not as effective as that of the SMFs. This is because when calculating the upper and lower envelops with cubic spline function in the sifting process of EMD, the extreme values at the two ends of the signal is uncertain, so that the envelopes are distorted, and the quality of performance was hurt. In addition, the SMF decomposed from the proposed method also has potential issues at the ends. That is, there will be much less samples to average close to the ends than in the middle of the time series.

As described in Eq.25, the Hilbert transform was performed on the SMFs to analyse the time-frequency characteristics of the signal. Fig. 4 compares the Hilbert spectrum of the proposed method and HHT. It can be observed that the proposed method outperformed the HHT in this case study. By comparing Fig. 3 and Fig. 4, it is found that although the results of IMFs and SMFs match well, the results in time-frequency spectrum are still quite different. To investigate the reasons for the differences, the components in Fig. 3 (a) are selected for comparison. First, Fourier transform was performed on the original component, the IMF and the SMF, respectively, and the results are shown in Fig. 5. The result of the original component 
(a) The Hilbert-Huang spectrum

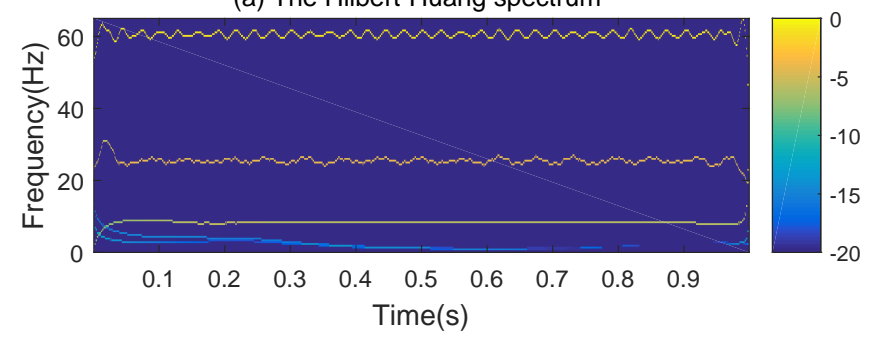

(b) The spectrum of the proposed method

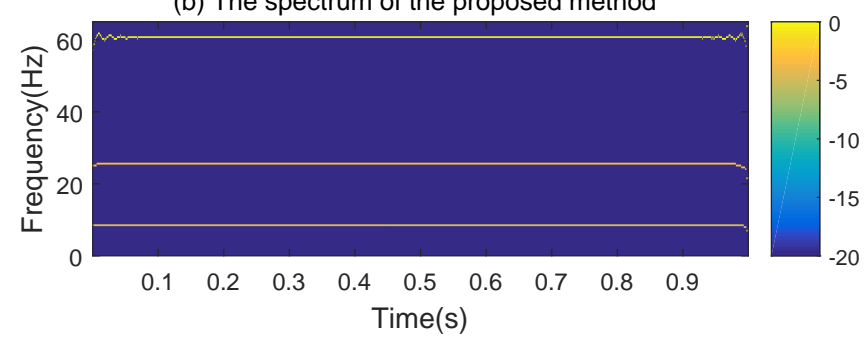

Fig. 4: Time-frequency analysis using (a) the HHT and (b) the proposed method.

is then used as a reference and is shown in Fig. 5 by the red line. It is seen that the result of SMF agrees well with the reference result. However, in addition to the mode of $60 \mathrm{~Hz}$, the result of IMF also has a component with $25 \mathrm{~Hz}$, which indicates that the mode-mixing problem still exists. Additionally, there are false modes present in the IMF result, such as the component with $20 \mathrm{~Hz}$. All these components lead to unsatisfactory time-frequency spectrum of EMD. So, improvements of the SMF-based method versus the HHT are achieved in the following three aspects: (1) avoiding the interaction between the components (i.e. mode mixing), (2) preventing false frequency components, and (3) reducing the endpoint effect.

\subsubsection{Scenario 2: Time-invariant signal with 12,13 and $15 \mathrm{~Hz}$}

The objective of this scenario was to evaluate the capacity of the proposed method to solve the modemixing problem. A set of frequencies (i.e. 12, 13, and $15 \mathrm{~Hz}$ in Table 1) was used for Eq.32 to simulate a signal with three close frequency components. The sampling interval $\Delta t$ was $0.001 \mathrm{~s}$, the number of data points $\mathrm{N}$ was 1000 , and $\Delta f$ was 0.1 . Fig. 6 compares the decomposition results of the IMFs and SMFs. It can be observed that the SMFs decomposed by the proposed method have acceptable consistency with the analytical results; however, the IMFs obtained from EMD failed to identify the original components owing to mode mixing. Fig. 7 (a) compares the waveforms of the IMF1 and the combination of all IMFs. It can be observed that the IMF1 is virtually the same as the combined signal. Fig. 7 (b) manifests the FFT spectrum of IMF1, where three frequencies (i.e. 12, 13, and $15 \mathrm{~Hz}$ ) can be observed. It is apparent 


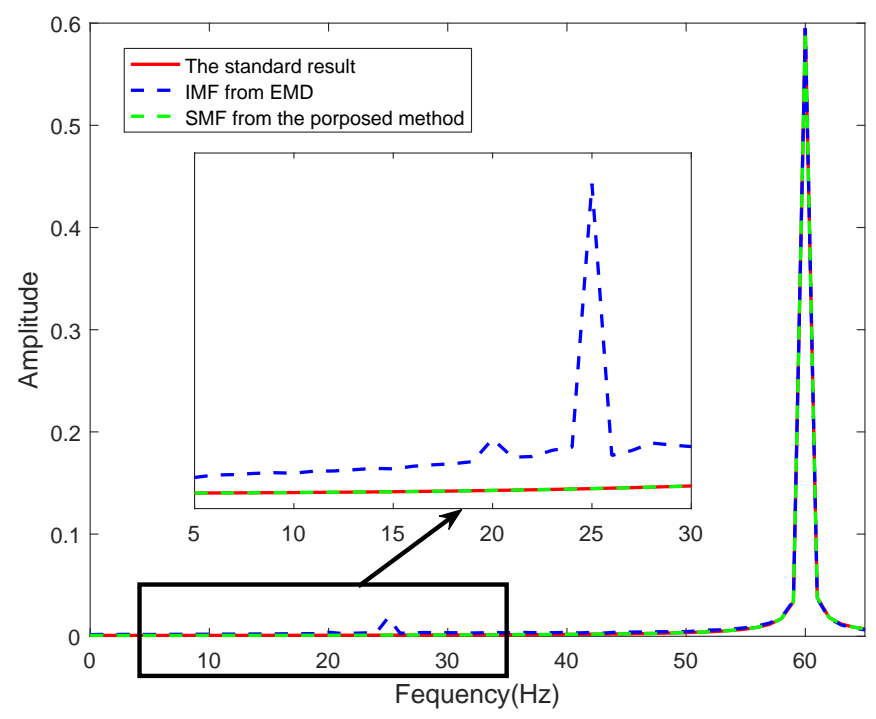

Fig. 5: A comparison of the first IMF and SMF in frequency domain.

that EMD did not separate the three frequency components into different IMFs; rather all were in IMF1. Conversely, the proposed method decomposed the three frequency components into three different SMFs. As indicated in Fig. 8, to identify close frequency components in this scenario, the mode mixing problem was solved effectively by the proposed method.

\subsection{Case study 2: Synthesized signal with slowly varying frequencies}

Notably, the frequencies in the above simulations were fixed. This is not realistic for offshore structures because of stochastic wave loading. To improve the practicality of the numerical simulation, a synthesised signal with 20 segments was used to evaluate the SMF decomposition. Again, Eq.32 was used to construct the synthesised signal. Each segment contained two frequency components (see Table 2): one frequency increased with time; the other decreased. In the simulation, each segment had 200 data points and the sampling frequency was $10 \mathrm{~Hz}$. Fig. 9 (a) displays the synthesised signal and Fig. 9 (b) indicates the theoretical instantaneous frequency of the signal. Moreover, to quantify the performance in terms of antialiasing, in the second scenario, Gaussian white noise was added to the synthesised signal.

\subsubsection{Scenario 1: Noise-free signal}

To analyse the time-frequency characteristic of the signal, WT, HHT and the proposed method were applied to the synthesised signal. WT is an linear method which use time and frequency shifts as two basic pa- 


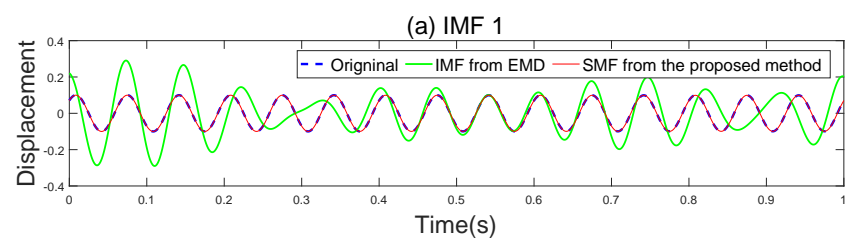

(b) IMF 2
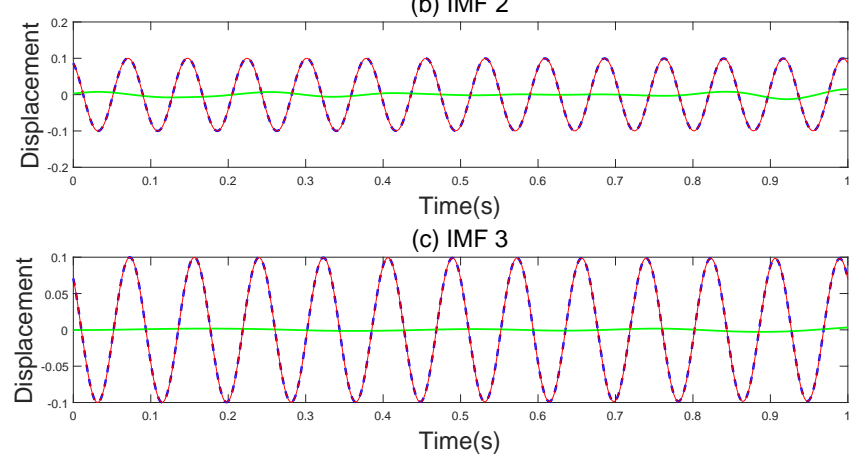

Fig. 6: Comparison between IMFs, SMFs and the original components: (a) IMF1, (b) IMF2 and (c) IMF3.
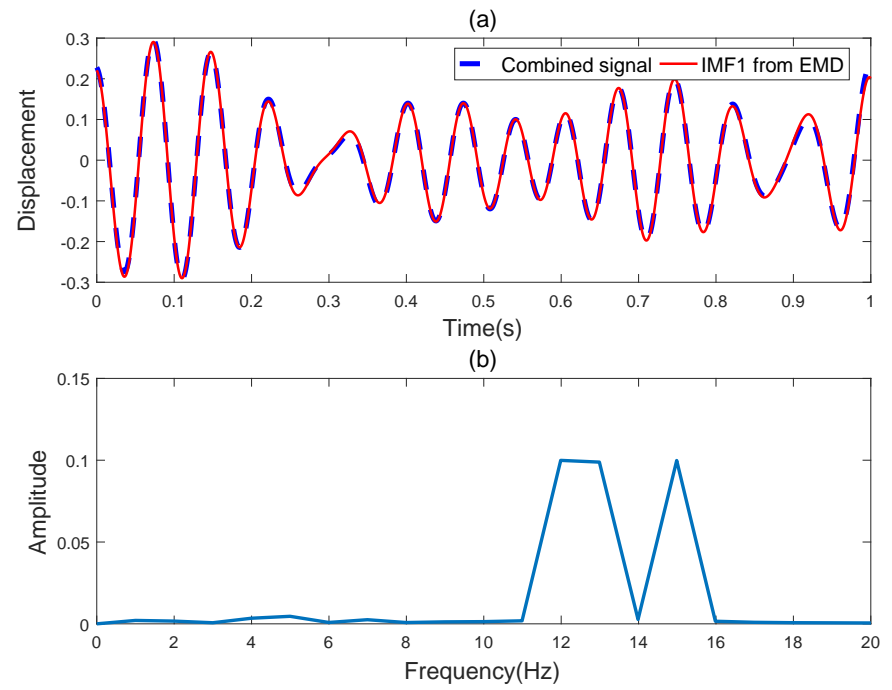

Fig. 7: (a) Comparison between IMF1 and the combined signal and (b) IMF1 obtained by EMD in the frequency domain. 

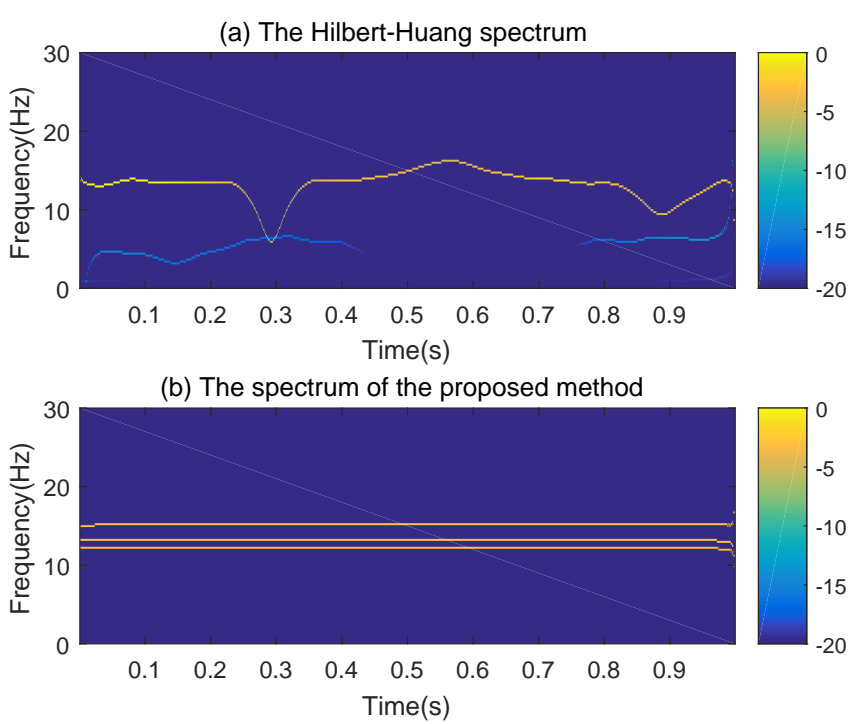

Fig. 8: Time-frequency analysis using (a) HHT and (b) the proposed method.
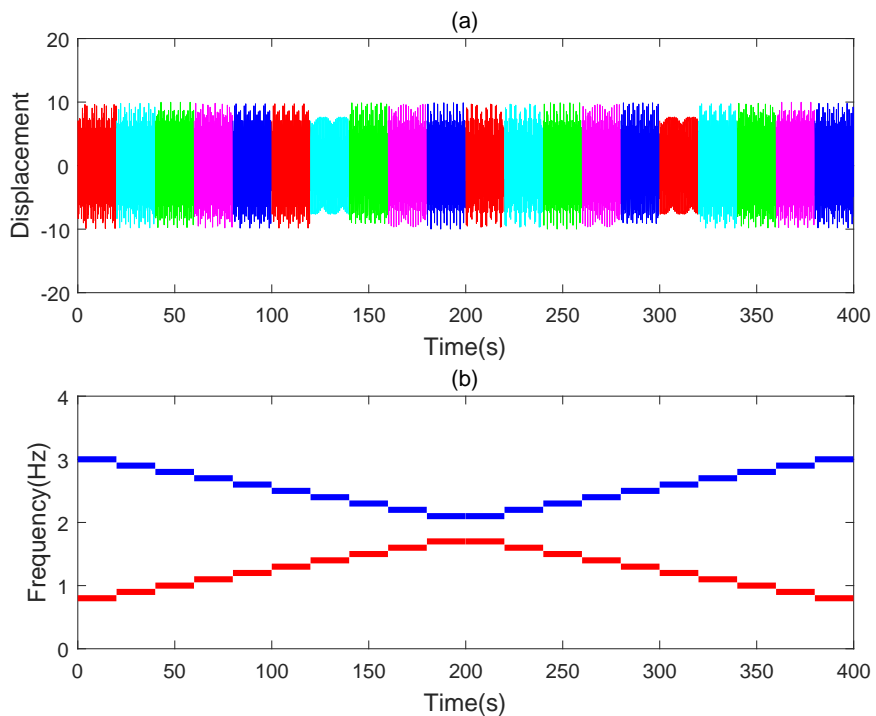

Fig. 9: Simulated signal with time-varying frequencies in (a) the time domain and (b) the frequency domain. 
Table 2: Frequencies set in Case study 2

\begin{tabular}{c|cccccccccc}
\hline$n$ & $f_{n}^{1}$ & $f_{n}^{2}$ & $f_{n}^{3}$ & $f_{n}^{4}$ & $f_{n}^{5}$ & $f_{n}^{6}$ & $f_{n}^{7}$ & $f_{n}^{8}$ & $f_{n}^{9}$ & $f_{n}^{10}$ \\
\hline 1 & 0.8 & 0.9 & 1.0 & 1.1 & 1.2 & 1.3 & 1.4 & 1.5 & 1.6 & 1.7 \\
\hline 2 & 3.0 & 2.9 & 2.8 & 2.7 & 2.6 & 2.5 & 2.4 & 2.3 & 2.2 & 2.1 \\
\hline $\mathrm{n}$ & $f_{n}^{11}$ & $f_{n}^{12}$ & $f_{n}^{13}$ & $f_{n}^{14}$ & $f_{n}^{15}$ & $f_{n}^{16}$ & $f_{n}^{17}$ & $f_{n}^{18}$ & $f_{n}^{19}$ & $f_{n}^{20}$ \\
\hline 1 & 1.7 & 1.6 & 1.5 & 1.4 & 1.3 & 1.2 & 1.1 & 1.0 & 0.9 & 0.8 \\
\hline 2 & 2.1 & 2.2 & 2.3 & 2.4 & 2.5 & 2.6 & 2.7 & 2.8 & 2.9 & 3.0 \\
\hline
\end{tabular}
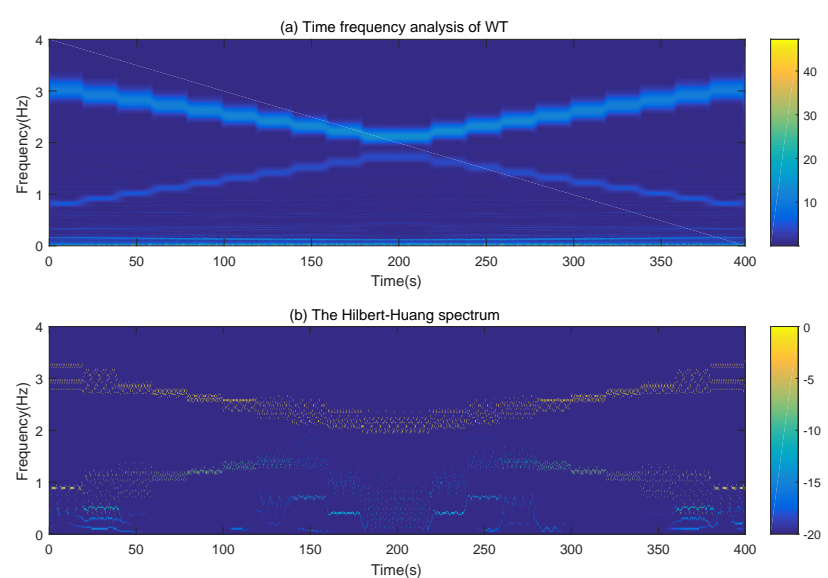

Fig. 10: Time-frequency analysis using (a) WT and (b) HHT methods.

rameters. These two parameters result in the formation of a rectangular block over the entire time-frequency domain, where the frequency and time determine the height and width of the window, respectively. Fig. 10 (a) depicts the time-frequency spectrum of WT. When address the signal, the Morlet wavelet was used as the mother wavelet function. From the results, we can observe that WT was a multiresolution method; the frequency resolution at the low-frequency part was superior. In fact, as WT is non-adaptive to a particular data series, and because of the limited length of the basic wavelet function, the results were influenced by leakages. This tends to lead to a time-frequency ridge pattern with thick and blurred lines, especially in the low-frequency range [38]. Moreover, as WT is a Fourier-based method, owing to the limited record length of the processed signal, the sampling frequency and signal frequency were not synchronised, and the problem of frequency leakage was inevitable, which caused false frequency components in the result $[39,40]$.

Fig. 10 (b) displays the time-frequency analysis result of the HHT. In the segments at both ends of the 


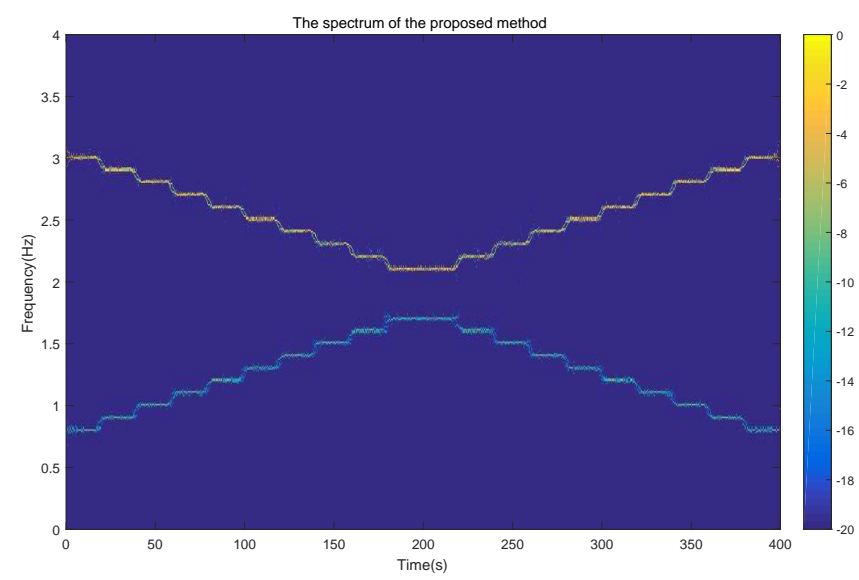

Fig. 11: Time-frequency analysis using the proposed method.

signal, EMD could achieve superior separation owing to the large difference between the two frequencies. However, as the two frequencies in the segments became closer, the decomposition performance decreased. When the difference between the two frequencies was less than $0.6 \mathrm{~Hz}$, the HHT failed to identify the two components. Moreover, there were numerous false frequency and noise components, which is because the two frequencies in each segment were too close, leading to mode mixing in the EMD decomposition process. Fig. 11 displays the analysis result of the proposed method. In the SMF decomposition process, the model order was set to 4 , the window size was 50 , and $\Delta f$ was 0.05 . It can be observed from Fig. 11 that the result of the proposed method had acceptable consistency with the theoretical result and it could process a signal having a time-varying frequency without mode mixing.

\subsubsection{Scenario 2: Noisy signal}

To demonstrate the effectiveness of the proposed method in processing noisy signals, in this scenario, Gaussian white noise was added in each segment of the signal in Fig. 9 (a). As the wave energy is weak in a real marine environment, the signal-to-noise ratio is relatively low. To simulate a real environment, herein, we consider the standard deviation of the Gaussian white noise as 0.6 and the average value as 4 , which is between the amplitudes of the two frequency components ( 2 and 8 ). First, the HHT was used to analyse the signal; the result is displayed in Fig. 12 (a). By comparing with Fig. 10 (b), we can observe that the HHT was extremely sensitive to noise, and the presence of noise seriously influenced the performance of the HHT.

By setting the model order to 20 , the window length to 50 and $\Delta f$ to 0.05 , the result obtained using the 

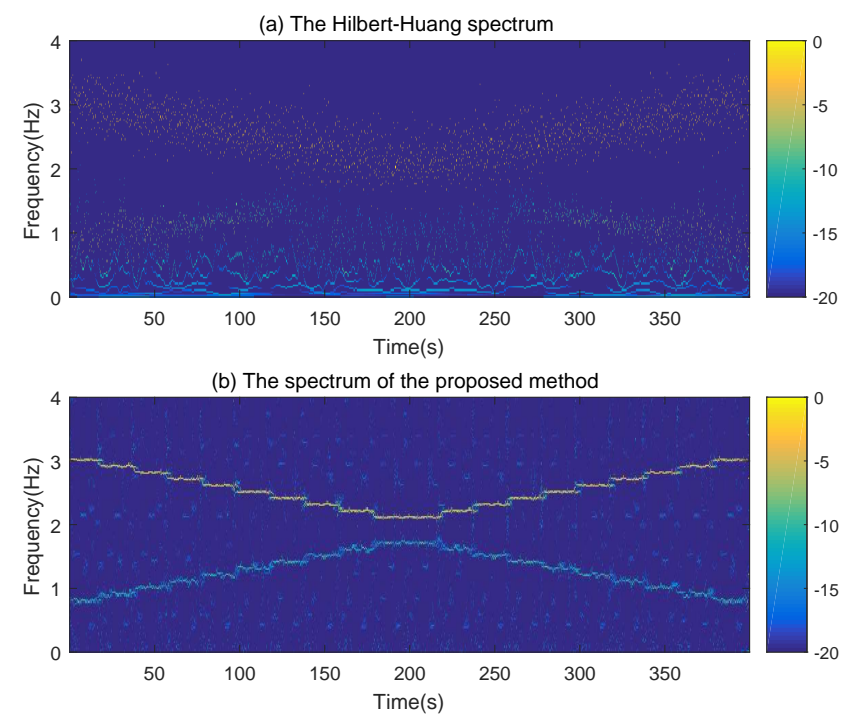

Fig. 12: Time-frequency analysis using (a) HHT and (b) the proposed method.

proposed method is displayed in Fig. 12 (b). The true frequency components are successfully decomposed and the changes of the noise components can be recognised. After decomposition, we can remove the two components from the synthesised signal. Fig. 13 illustrates the noise between 180 and 200s after removing the two real components; the blue line is the original added noisy component. By comparison, we can find that, owing to the mode mixing in the HHT method, part of the noise components were recognised as the true component of the synthesised signal. Furthermore, we can observe that the proposed method was not sensitive to the noise; the results are consistent with the original noise. Especially when the signal-to-noise ratio was low, the real components could be separated well. Hence, this approach is more suitable for the vibration signal analysis of structures under a complex marine environment.

\section{Case study 3: Experimental validation}

In this case study, the performance of the proposed method was evaluated using experimental measurements. The experimental data were acquired from a high-mode vortex-induced vibration (VIV) experiment sponsored by the Norwegian Deepwater Programme (NDP). Based on the settings, a hydrodynamics testing program for a riser with a high length-to-diameter ratio of 1400 (i.e., $38 \mathrm{~m}$ in length and $0.027 \mathrm{~m}$ in diameter) was conducted. The riser was dragged at a constant speed in a water tank to simulate a uniform/sheared current environment. The micro bending strain and acceleration sensors were installed in the in-line (IL) 


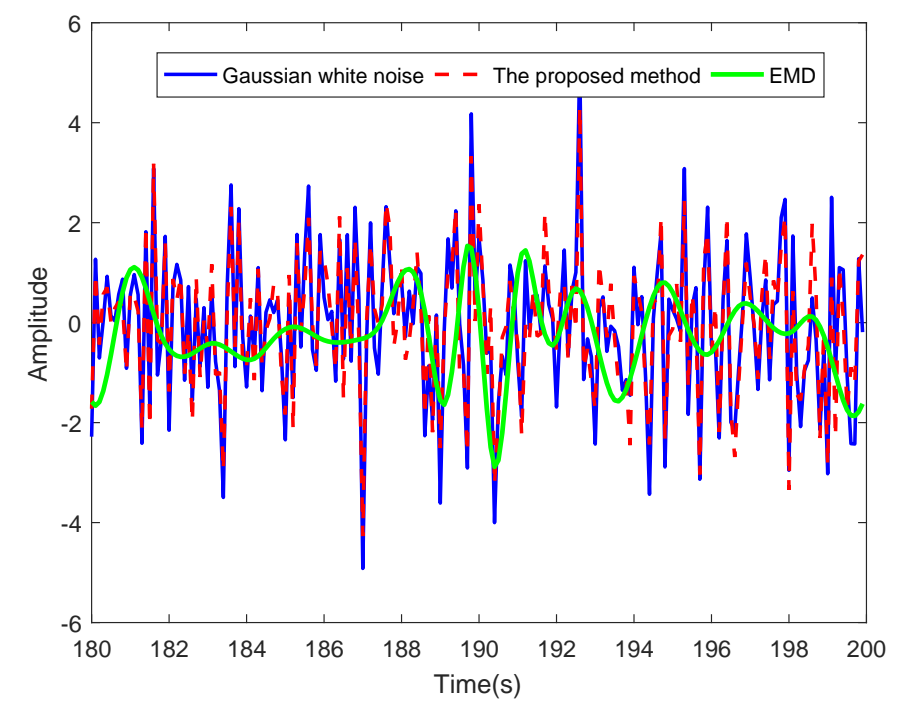

Fig. 13: Comparison of noise after decomposition.

and cross-flow (CF) directions along the riser to collect the experimental data. More details can be found in references [41, 42]. As mentioned in [43, 44], the motions between the CF and IL were coupled, and the frequency of the IL response was twice that of CF. This difference allowed us to verify the correctness of the time-frequency analysis results.

In the present study, the acceleration data set at $\triangle / L=0.1314$ of the IL directions from Test 2350 were used. The sheared current profiles in these data sets corresponded to a towing speed of $0.7 \mathrm{~m} / \mathrm{s}$. Fig. 14 displays the acceleration signal in the time domain and frequency domain. Fig. 15 compares the time-frequency analysis results of WT, HHT, and the proposed method. In the result obtained using WT, as displayed in Fig. 15 (a), three clear frequency components can be observed; however, owing to the limitation of the window in WT, the result cannot maintain the same resolution over the entire frequency range. As the frequency increases, the resolution becomes increasingly worse. Fig. 15 (b) displays the time-frequency analysis result of the HHT method. A large energy strip concentrated near $6 \mathrm{~Hz}$, with energy near $13 \mathrm{~Hz}$, was barely observed. Moreover, owing to mode mixing, numerous false frequency and noise components appeared in the low frequency part. Hence, the decomposition performance of the HHT was poor and could not correctly discover the true feature frequencies in the VIV data set.

Fig. 15 (c) depicts the analysis result of the SMF-based method. In the analysis, the window length was 300 , the model order was 120 , and $\triangle f$ was 0.05 . From the time-frequency spectrum, two clear frequency components at $6.77 \mathrm{~Hz}$ and $13.55 \mathrm{~Hz}$ can be observed, which satisfies the relationship of two times and 
(a)

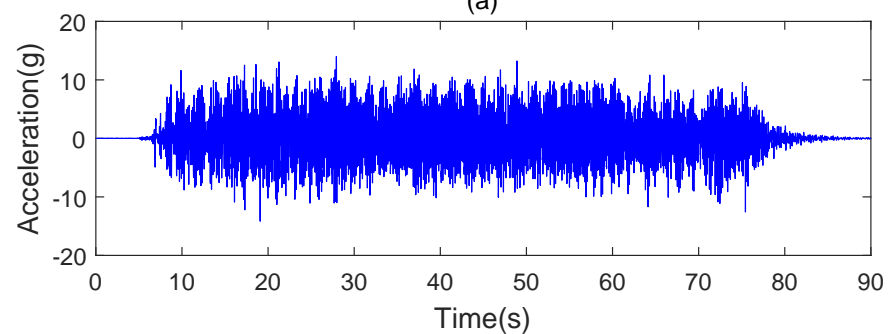

(b)

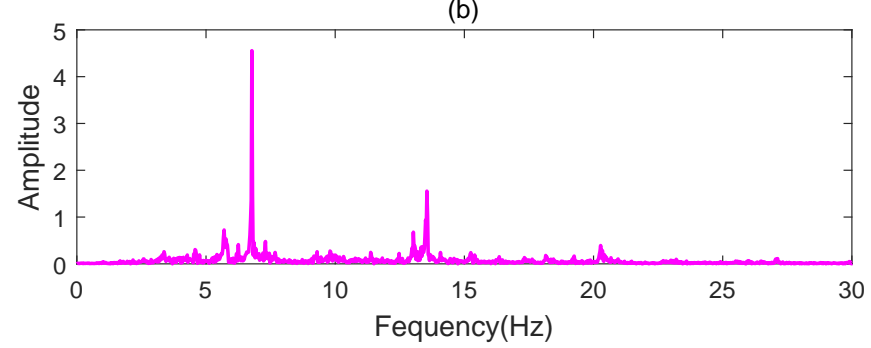

Fig. 14: Acceleration signal of IL in the time and frequency domain from Test2350 of NDP high-mode text.

is consistent with the experimental results. In addition, a third frequency component at $20.31 \mathrm{~Hz}$ can be observed. This was an unexpected combination of the two frequency components called the 'non-linear interaction frequency', which is a new phenomenon first reported by Rao et al. in 2015 [45]. However, in the HHT, we could not observe this phenomenon. Furthermore, although noise and disturbance components were observed in Fig. 15 (c), they were considerably less influential on the main energy strips when compared with the result in Fig. 15 (b).

\section{Case study 4: Field data gathered from offshore wind turbine structures}

In this case study, the proposed method was verified using the field data collected from offshore wind turbine structures in an offshore wind farm $32 \mathrm{~km}$ from the shore in the Jiangsu Province of China. Accelerometer sensors (Model 4630 and Model 4083A) were installed via the magnetic bases on the wind turbine structures to measure the structural dynamic responses. The CS $5008 \mathrm{~N}$ acquisition instrument (16 channels) manufactured by imcAccess of Germany was used to store the structural vibration data. In this case, two sets of measured data were used for the analysis, one from a foundation before the wind turbine was installed and the other from a foundation with the wind turbine under operation. 

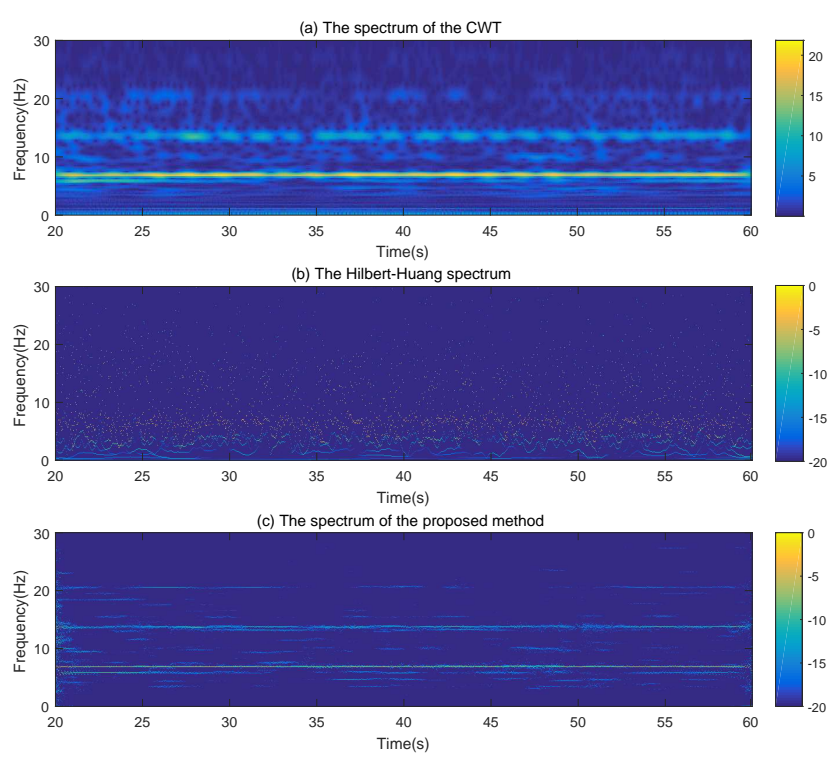

Fig. 15: Time-frequency analysis using (a) WT, (b) HHT, and (c) the proposed method.

\subsection{Analysis of structure without the turbine}

Fig. 16 displays an image of the foundation of the wind turbine whose first-order design frequency is $2.1571 \mathrm{~Hz}$. Five sensors were arranged in order from the top of the foundation; the interval of the arrangement was $2.5 \mathrm{~m}$. The sampling frequency was $200 \mathrm{~Hz}$ and the field test lasted for $30 \mathrm{~min}$. Here, the signal in the $x$ direction of the second sensor was selected for the time-frequency analysis. Fig. 17 (a) displays the waveform of the signal measured by the sensor. The signal between 420 and $570 \mathrm{~s}$ (displayed in Fig. 17 (b)) was used to perform the time-frequency analysis. Owing to the weak wave energy and significant stiffness, the true mode was difficult to be excited. Hence, herein, a ship collision was chosen to increase the impact energy. This is also reflected in Fig. 17 (b). When the impact occurred, the amplitude was magnified. Fig. 18 compares the analysis results of the HHT and the proposed method.

The result of the HHT is displayed in Fig. 18 (a). Corresponding to the selected signal in the time domain, energy appears when the impact occurred. Strong energy occurred near $2.2 \mathrm{~Hz}$, and its intensity varied with time. However, severe mode mixing caused aliasing owing to the influence of noise in the marine environment. Fig. 18 (b) displays the SMF decomposed result. In the analysis, the model order was 450, the window length was 1000 , and $\Delta f$ was 0.01 . From Fig. 18 (b), it can be observed that the first natural frequency of the structure was near $2.2 \mathrm{~Hz}$ which also consistent with the design frequency of the 


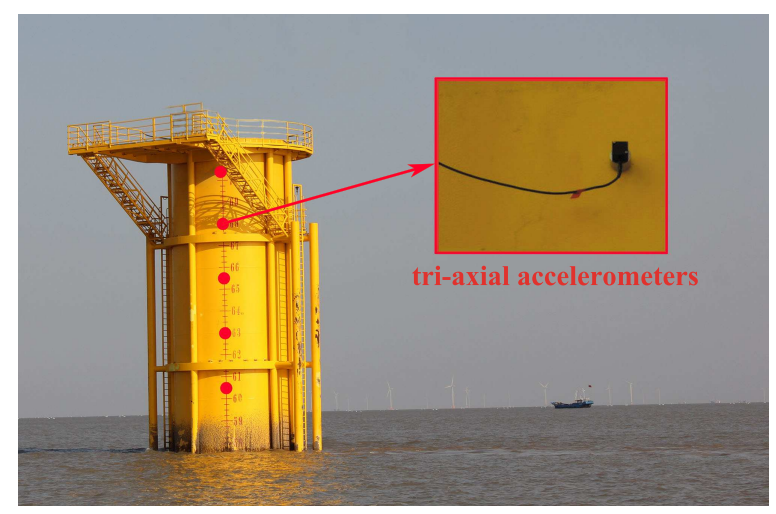

Fig. 16: Test offshore wind turbine foundation.

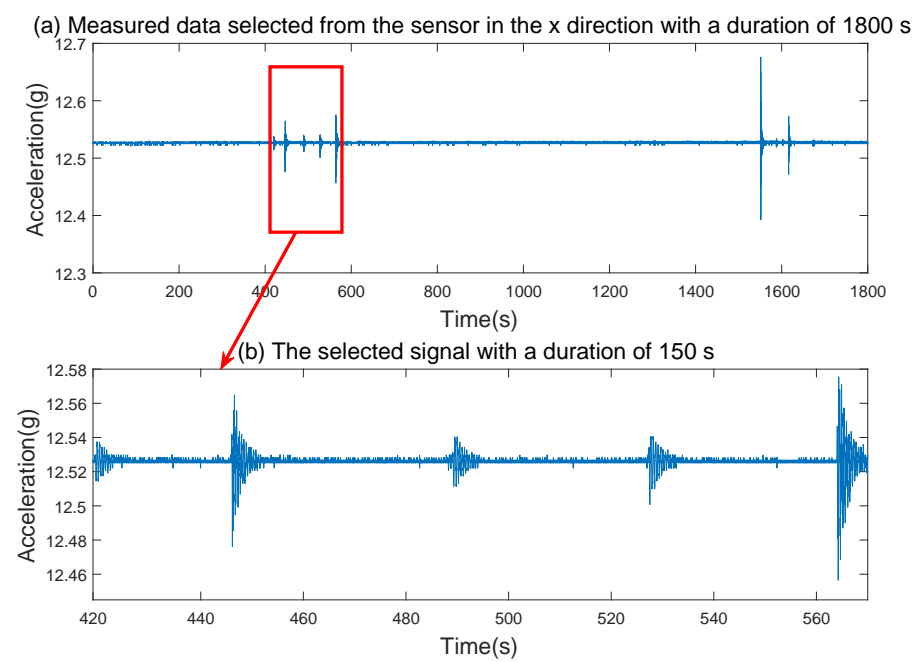

Fig. 17: (a) Measured data selected from sensor in the $x$ direction with the duration of $1800 \mathrm{~s}$; (b) The selected signal with a duration of $150 \mathrm{~s}$.

structure. Owing to the weak energy of the wave, the natural frequency of the structure could not be excited in the response signal. Also, this result confirms the discontinuous nature of frequencies in the vibration response from an offshore wind turbine structure. Fig. 18 (b) also displays the time-varying frequency characteristics of the signal from Fig. 17 (b). Based on the time-frequency analysis results, an appropriate time period can be determined for the subsequent modal analysis of the structural vibration.

\subsection{Analysis of an operating offshore wind turbine}

Five tri-axial accelerometer sensors were used to measure the wind turbine vibration signal. The sensors were installed separately from the bottom to the top at the heights of $0,16.3,42.7,59.4$, and $73.4 \mathrm{~m}$, as 

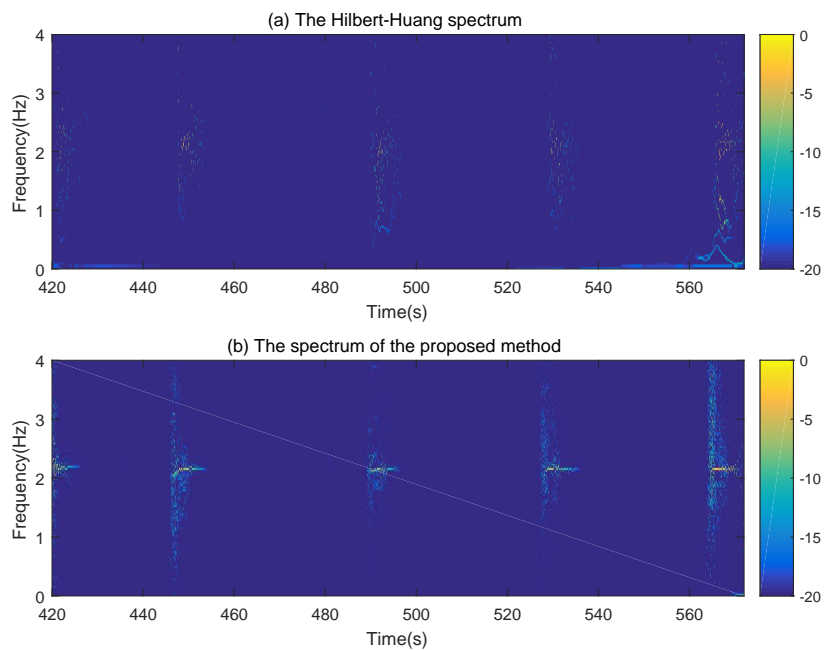

Fig. 18: Time-frequency analysis using (a) HHT and (b) the proposed method.

indicated in Fig. 19. The sampling frequency was $200 \mathrm{~Hz}$ and the recording time was $60 \mathrm{~min}$. The data measured by the second sensor in the horizontal direction were used for the analysis. Fig. 20 (a) displays the original vibration signal of the wind turbine and Fig. 20 (b) displays the selected signal with a duration of $50 \mathrm{~s}$ (from $1750 \mathrm{~s}$ to $1800 \mathrm{~s}$ ). The time-frequency result of the HHT is displayed in Fig. 21 (a). As indicated in the figure, the most significant frequency was near $0.2 \mathrm{~Hz}$, but fluctuation is apparent. Owing to the influence of similar frequency components, the mode mixing was severe and it was difficult to discover any useful information regarding the wind turbine dynamics using the HHT.

Fig.21 (b) displays the SMF decomposed result. In the analysis, the model order was 300, the window length was 1000 , and $\Delta f$ was 0.01 . It can be observed from Fig. 21 (b) that the fundamental frequency was $0.3 \mathrm{~Hz}$. Moreover, there was weak energy appearing near $1.7 \mathrm{~Hz}$. These two frequencies were consistent with the designed frequencies of this offshore wind turbine, whose first and second-order design frequencies were $0.3056 \mathrm{~Hz}$ and $1.7296 \mathrm{~Hz}$. Owing to the weak wave energy and effects of noise, the excitation for the fundamental frequency of the structure was intermittent. A major difference between the operating structure and foundation in Section 6.1 is that the loads/excitations generated from the turbine rotor have a significant influence on the structural dynamics. This effect is mainly reflected in the components appearing in multiple frequencies displayed in Fig. 21 (b), which increase the difficulty of recognition for the natural frequencies of the offshore wind turbines [46]. By comparing the results of HHT and SMF decomposition, it is observed that the proposed method outperformed the HHT in terms of the analysis accuracy and mode 


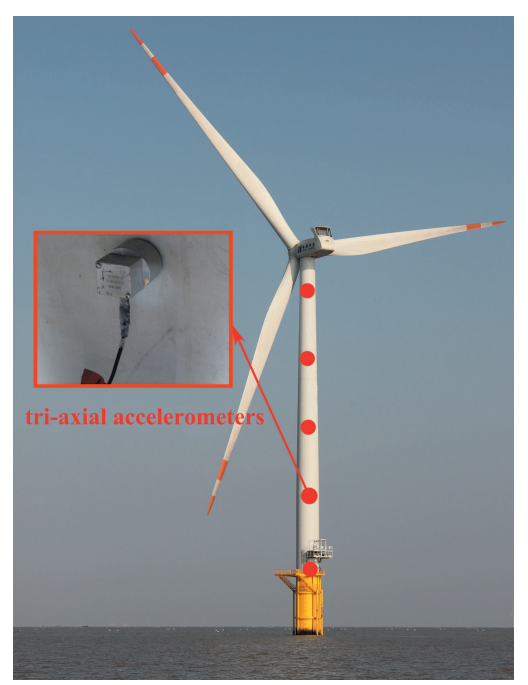

Fig. 19: Test offshore wind turbine.
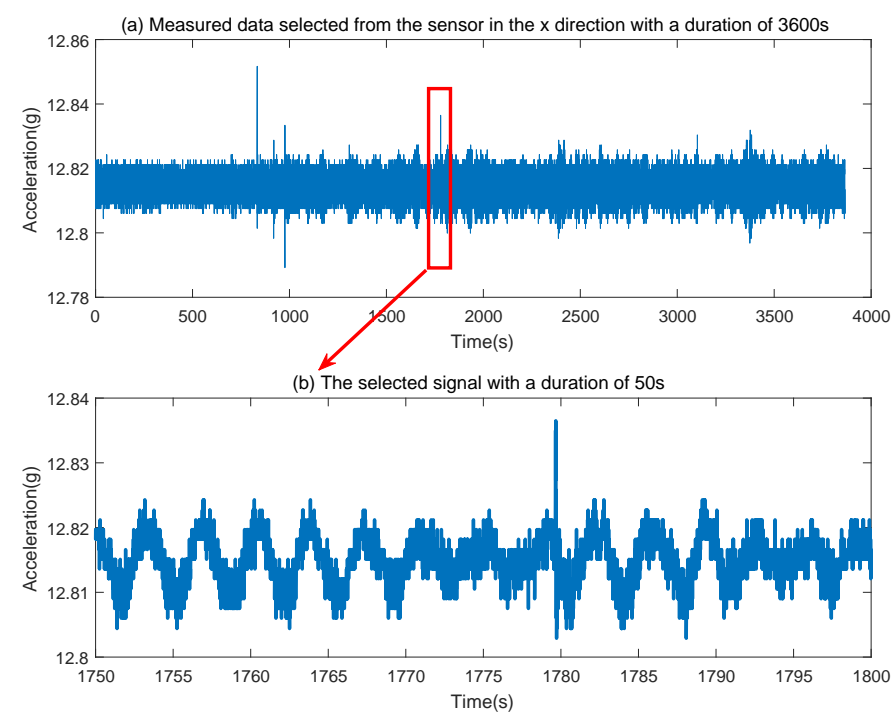

Fig. 20: (a) Measured vibration signal with the duration of 3600s from the operating offshore wind turbine; (b) The selected signal with a duration of $50 \mathrm{~s}$. 

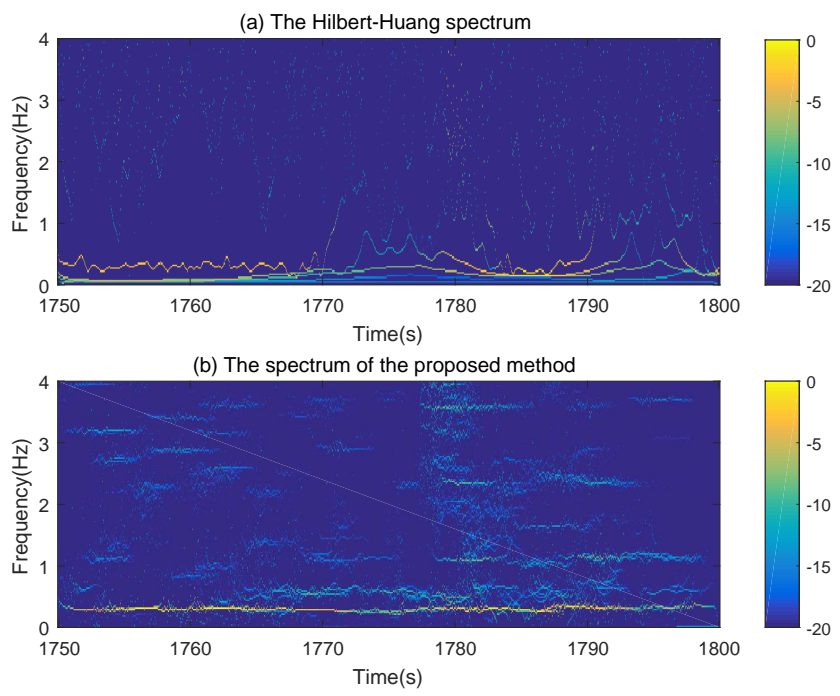

Fig. 21: Time-frequency analysis using (a) HHT and (b) the proposed method.

mixing.

\section{Conclusions}

Based on the energy recollection and Hilbert transform, a time-frequency analysis method was proposed to solve the difficulties of similar frequency components in offshore wind turbines. In this approach, a sliding window was used to segment the vibration signal. And inside each window, a state-space model was introduced to improve the accuracy of the complex exponential decomposition. The moving step was set to be one. Then, by implementing the energy recollection in a special energy gridding, the SMF, which was equal to the IMF in the traditional approach, was obtained. Unlike tradition IMF, which is empirical, the proposed method obtained the expression of the SMF through a more rigorous mathematical derivation and could overcome the mode mixing phenomenon.

The proposed method was applied to four examples including two numerical examples, a VIV experiment, and an offshore wind turbine in the field. The first numerical example was a signal with three time-invariant frequency components, where two scenarios, frequency components with a considerable difference and similar frequency components were studied. The results demonstrated that the mode mixing phenomenon could be reduced significantly by the proposed method. The second numerical example was one signal with time-varying frequency components that changed slowly. The results indicated that the proposed method could analyse the time-varying signal well and avoid non-relevant frequency components. 
Moreover, experimental data from the VIV test by the NDP were used in another example. The experimental results indicated that the proposed method could effectively overcome mode-mixing in the measured data. Finally, two groups of sea-test data were used. One data set was from an offshore wind turbine foundation and the other was from an operating offshore wind turbine. The results confirmed that the time-varying characteristics could be captured in the time-frequency spectrum and the proposed method could obtain a sharper and superior performance.

\section{Acknowledgements}

The authors acknowledge the financial support of the National Natural Science Foundation of China (U1806229), the Taishan Scholars Program of Shandong Province and the generous permission by the Norwegian Deepwater Program authors to use their experimental data.

\section{Reference}

[1] Gao Z, Moan T, Wan L, Michailides C. Comparative numerical and experimental study of two combined wind and wave energy concepts. J Ocean Eng Sci 2016;1(1):36-51.

[2] Brennan F. Risk Based Maintenance for Offshore Wind Structures. Proc CIRP 2013;11:296-300.

[3] Dong XF, Lian JJ, Wang HJ, Yu TS, Zhao Y. Structural vibration monitoring and operational modal analysis of offshore wind turbine structure. Ocean Eng 2018;150:280-297.

[4] Seyr H, Muskulus M. Safety Indicators for the Marine Operations in the Installation and Operating Phase of an Offshore Wind Farm. Energy Proc 2016;94:72-81.

[5] Ren ZR, Jiang ZY, Skjetne R, Gao Z. Development and application of a simulator for offshore wind turbine blades installation. Ocean Eng 2018;15:380-395.

[6] Sclavounos PD. Karhunen-Loeve Representation of Stochastic Ocean Waves. Proc R Soc A-Math Phys Eng 2012;468:25742594.

[7] Iliopoulos A, Shirzadeh R, Weijtjens W, Guillaume P, Hemelrijck VD, Devriendt C. A modal decomposition and expansion approach for prediction of dynamic responses on a monopile offshore wind turbine using a limited number of vibration sensors. Mech Syst Signal Process 2016;68-69:84-104.

[8] Owem JS, Eccles BJ, Choo BS, Woodings MA. The application of auto-regressive time series modelling for the timefrequency analysis of civil engineering structures. Eng Struct 2001;23(5):521-536.

[9] Sejdic E, Djurovic L, Jiang J. Time-frequency feature representation using energy concentration: An overview of recent advances. Digit Signal Prog 2009;19(1):153-183.

[10] Sejdic E, Orovic I, Stankovic S. Compressive sensing meets time-frequency: An overview of recent advances in timefrequency processing of sparse signals. Digit Signal Prog 2018;77:22-35.

[11] Boashash B. Time Frequency Signal Analysis and Processing: A Comprehensive Reference (2nd). Elsevier, Amsterdam 2016. 
[12] Neild SA, McFadden PD, Williams MS. A review of time-frequency methods for structural vibration analysis. Eng Struct 2003;25(6):713-728.

[13] Xing JT, Price WG. The theory of non-linear elastic ship-water interaction dynamic. J Sound Vibr 2000;230(4):877-914.

[14] Feng ZP, Liang M. Fault diagnosis of wind turbine planetary gearbox under nonstationary conditions via adaptive optimal kernel time-frequency analysis. Renew Energy 2014;66:468-477.

[15] Gabor D. Theory of communications. J IEEE 1946;93(3):429-457.

[16] Grossmann A, Morlet J. Transform asociated with square intergrable group representation, part i. J Math Phys 1985;26:24732479.

[17] Dekys V, Kalman P, Hanak P, Novak P, Stankovicova Z. Determination of vibration sources by using STFT. Procedia Eng 2017;177:496-501.

[18] Yu Y, Shenoi RA, Zhu HB, Xia LJ. Using wavelet transforms to analyze nonlinear ship rolling and heave-roll coupling. Ocean Eng 2006;33(7):912-926.

[19] Huang NE, Shen Z, Long SR, Wu MC, Shih HH, Zheng QN. The empirical mode decompositiom and the Hilbert spectrum for nonlinear and non-stationary time series anaysis. Proc R Soc A 1998;454:903-995.

[20] Huang NE, Shen Z, Long SR. A new review of nonlinear water waves: the Hilbert spectrum. Annu Rev Fluid Mech 1999;31:417-457.

[21] Dong, XF, Lian JJ, Wang, HJ. Vibration source identification of offshore wind turbine structure based on optimized spectral kurtosis and ensemble empirical mode decomposition. Ocean Eng 2019;172:199-212.

[22] Peng ZK, Tse PW, Chu FL. An improved Hilbert-Huang transform and its application in vibration signal analysis. J Sound Vibr 2005;286:187-205.

[23] Rato TR, Ortigueira MD, Batista AG. On the HHT, its problems, and some solutions. Mech Syst Signal Process 2008;22:1374-1394.

[24] Wu Z, Huang NE. Ensemble empirical mode decomposition: a noise-assisted data analysis method. Adv Adapt Data Anal 2009;1:1-41.

[25] Huang TL, Lou ML, Chen HP, Wang NB. An orthogonal Hilbert-Huang transform and its application in the spectral representation of earthquake accelerograms. Soil Dyn Earthq Eng 2018;104:378-389.

[26] Prony R. Essai experiment et analytique: sur les lois de la dilatabilite des fluids elastiques et sur celles de la force expansive de la vapeur del'eau et de la vapeur de l'alkool, a differentes temperatures (in French). J de l'Ecole Polytechnique 1795;1(2):2476.

[27] Hu S-LJ, Yang WL, Li HJ. Signal decomposition and reconstruction using complex exponential models. Mech Syst Signal Process 2013;40:421-438.

[28] Tian Z, Liu FS, Zhou L, Yuan CF. Fluid-structure interaction analysis of offshore structures based on separation of transferred responses. Ocean Eng 2020;195:106598.

[29] Liu FS, Fu Q, Tian Z. A Laplace-domain method for motion response estimation of floating structures based on a combination of generalised transfer function and partial fraction. Ships Offshore Struc 2019;DOI:10.1080/17445302.2019.1687145.

[30] Carson J, Fry T. Variable frequency electric circuit theory with application to the theory of frequency modulation. Bell Labs Tech J 1937;16:513-540.

[31] Van der Pol B. The Fundamental Principles of Frequency Modulation. Proc IEEE 1946;93(3):153-158. 
[32] Kreyszig EO, Kreyszig H, Norminton EJ. Advanced Engineering Mathematics, 10th edition. John Wiley Sons, Inc., Hoboken, NJ, USA 2011.

[33] Polking JC, Boggess A, Arnold D. Differential Equations. Pearson Prentice Hall 2006.

[34] Rilling G, Flandrin P, Goncalves P. On empirical mode decomposition and its algorithms. IEEE-EURASIP 2003;1:1-5.

[35] Xu GL, Wang XT, Xu XG, Zhou LJ. Improved EMD for the analysis of FM signals. Mech Syst Signal Process 2012;33:181196.

[36] Yang ZH, Zhang Q, Zhou F, Yang LH. Hilbert spectrum analysis of piecewise stationary signals and its application to texture classification. Digit Signal Process 2018;82;1-10.

[37] Liu FS, Li HJ, Wang WY, Li W, Wang B. Frequency variation and sensor contribution assessment: Application to an offshore platform in the South China Sea. J Sound Vibr 2015;337:218-232.

[38] Wang C, Ren WX, Wang ZC, Zhu HP. Instantaneous frequency identification of time-varying structures by continuous wavelet transform. Eng Struct 2013;52:17-25.

[39] Antoni J. Leakage-free identification of FRF's with the discrete time Fourier transform. J Sound Vib 2006;294;(4-5):9811003.

[40] Diao RP, MENG QF. Frequency estimation by iterative interpolation based on leakage compensation. Measurement 2015;59:44-50

[41] Braaten H, Lie H. NDP riser high mode VIV tests main report. Main report number 512394.00.01. Norwegian Marine Technology Research Institute, 2004.

[42] Trim AD, Braaten H, Lie H, Tognarelli MA. Experimental investigation of vortex induced vibration of long marine risers. J Fluids Struct 2005;21:335-361.

[43] Vandiver JK, Ma LX, Rao ZB. Revealing the effects of damping on the flow-induced vibration of flexible cylinders. J Sound Vibr 2018;433:29-54

[44] Liu YC, Liu FS, Wang EH, Xiao Q, Li L. The effect of base column on vortex-induced vibration of a circular cylinder with low aspect ratio. Ocean Eng 2020;196:106822.

[45] Rao ZB, Vandiver JK, Jhingran V. Vortex induced vibration excitation competition between bare and buoyant segments of flexible cylinders. Ocean Eng 2015;94:186-198.

[46] Liu FS, Gao SJ, Han HW, Tian Zhe, Liu P. Interference reduction of high-energy noise for modal parameter identification of offshore wind turbines based on iterative signal extraction. Ocean Eng 2019;183:372-383. 